\title{
Podocyte-specific Rac1 deficiency ameliorates podocyte damage and proteinuria in STZ-induced diabetic nephropathy in mice
}

Zhimei Lv', Mengsi Hư ${ }^{1}$ Minghua Fan², Xiaobing Li ${ }^{3}$, Jiangong Lin ${ }^{1}$, Junhui Zhen ${ }^{4}$, Ziyang Wang ${ }^{1}$, Haijun Jin ${ }^{5}$ and Rong Wang ${ }^{1}$

\begin{abstract}
Activation of Ras-related C3 botulinum toxin substrate 1 (Rac1) has been implicated in diverse kidney diseases, yet its in vivo significance in diabetic nephropathy (DN) is largely unknown. In the present study, we demonstrated a podocyte-specific Rac1-deficient mouse strain and showed that specific inhibition of Rac1 was able to attenuate diabetic podocyte injury and proteinuria by the blockade of Rac1/PAK1/p38/ $\beta$-catenin signaling cascade, which reinstated the integrity of podocyte slit diaphragms (SD), rectified the effacement of foot processes (FPs), and prevented the dedifferentiation of podocytes. In vitro, we showed Rac1/PAK1 physically bound to $\beta$-catenin and had a direct phosphorylation modification on its C-terminal Ser675, leading to less ubiquitylated $\beta$-catenin, namely more stabilized $\beta$-catenin, and its nuclear migration under high-glucose conditions; further, p38 activation might be responsible for $\beta$-catenin nuclear accumulation via potentiating myocyte-specific enhancer factor $2 C$ (MEF2c) phosphorylation. These findings provided evidence for a potential renoprotective and therapeutic strategy of cellspecific Rac1 deficiency for DN and other proteinuric diseases.
\end{abstract}

\section{Introduction}

Diabetic nephropathy (DN) is one of the leading causes for chronic kidney diseases ${ }^{1}$. Podocytes are terminally differentiated epithelial cells of the glomerulus, essential for the maintenance of an intact glomerular filtration barrier (GFB), and damaged podocytes are a key contributor to the onset of proteinuria and the progression of $\mathrm{DN}^{2-4}$. To date, mechanisms that govern diabetic podocyte damage and kidney injury remain poorly understood.

Rac1, a member of Rho family small GTPases, is a multi-functional molecule implicated in various cellular

\footnotetext{
Correspondence: Rong Wang (sdwangrong@sina.cn)

${ }^{1}$ Department of Nephrology, Shandong Provincial Hospital Affiliated to

Shandong University, Jinan, China

${ }^{2}$ Department of Obstetrics and Gynecology, The Second Hospital of Shandong University, Jinan, China

Full list of author information is available at the end of the article Edited by J. Chipuk
}

processes involving cell adhesions, proliferation, and plasticity $^{5}$. Abnormal Rac1 signaling is involved in ROS production and inflammatory responses and reportedly linked to a number of debilitating human diseases, including cancer, diabetes, and kidney disorders ${ }^{5-8}$. Kolavennu et al. demonstrated in vivo targeting RhoA signaling, another pivotal member of Rho GTPases, could ameliorate albuminuria in a rodent model of diabetes via downstream signaling ROCK ${ }^{9}$. And we previously showed in vitro that Rac1 interference was protective against high-glucose (HG)-induced podocyte damage ${ }^{10}$, hence we postulated that manipulating Rac1 expression might as well be of a curative potentiality in vivo. Notably, systemic $\mathrm{KO}$ of Rac1 would lead to embryonic lethality in mice due to germ-layer formation defects ${ }^{11,12}$. Thus in the present study, we described a podocyte-specific Rac1-deficient mouse strain and generated diabetes models in these 


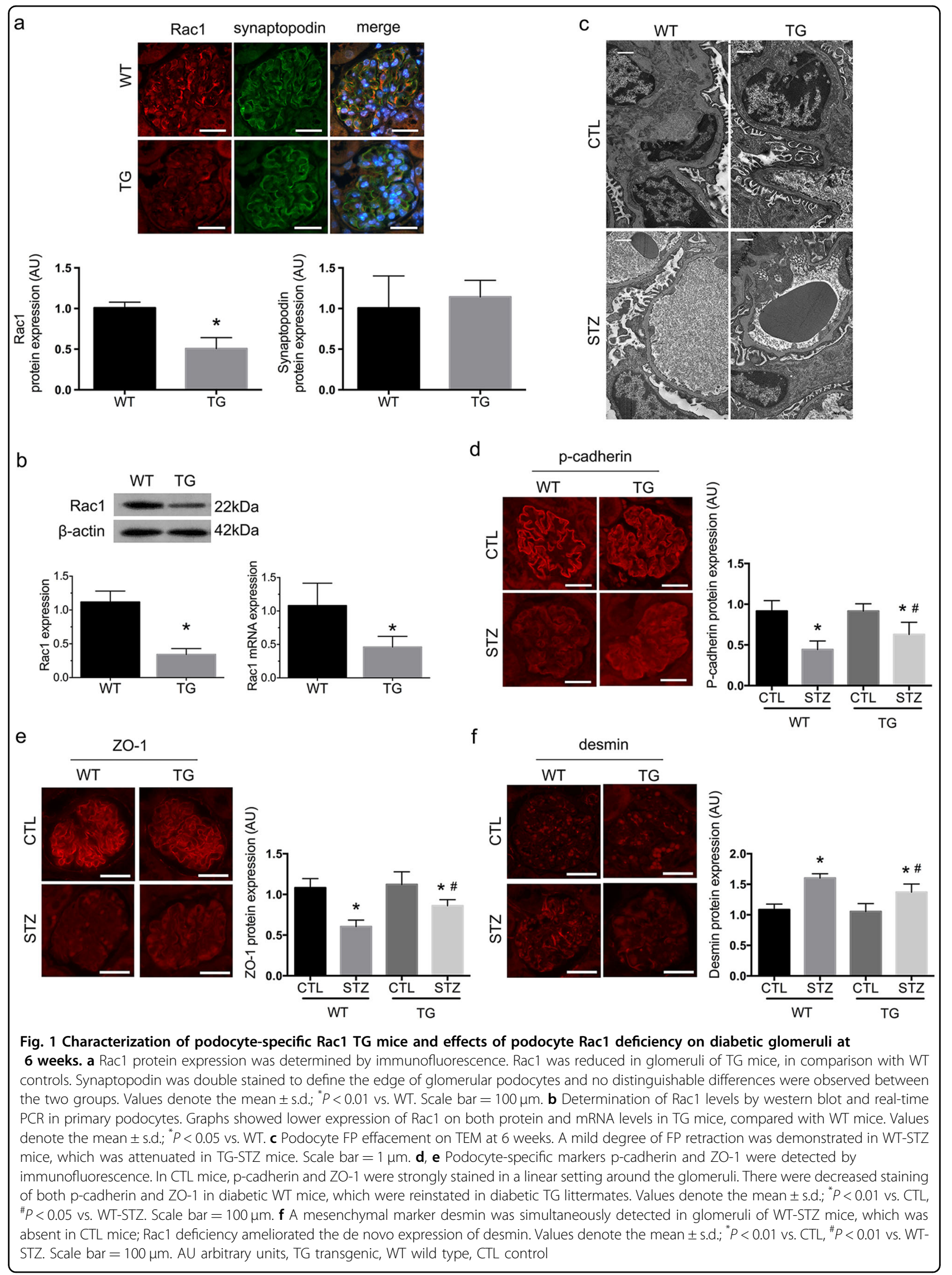


mice, aiming to uncover a renoprotective and therapeutic role of cell-specific Rac1 deficiency in DN and related podocyte damage.

Among many factors implicated in the pathogenesis of DN, p38 MAPK (p38), which belongs to MAPK family, is typically involved in diabetes as a critical mediator of inflammatory reactions and mitochondrial malfunction ${ }^{13}$. Hyperphosphorylated p38 is found in renal proximal tubular epithelial cells (PTECs) and contributes to their epithelial-mesenchymal transition (EMT) ${ }^{13,14}$. Aberrant p38 phosphorylation was also correlated with the modulation of podocyte cytoskeletal dynamics ${ }^{15}, 16$. Additionally, p38 could be activated by PAK1, a major downstream target of Rac1, as reported in several cancer cell lines and tracheal smooth muscle cell ${ }^{17}{ }^{18}$. However, interplays between Rac1/PAK1 and p38 in diabetic podocytes and how would these interactions contribute to podocyte damage and proteinuria is not fully clarified.

It was reported that Rac1 activation controlled nuclear localization of $\beta$-catenin, a key intracellular signal transducer involved in kidney fibrosis, during canonical Wnt signaling, depending on phosphorylation at its Ser191 and Ser605 ${ }^{19}$. In our previous study, Rac1/PAK1 activation was sufficient to trigger enhanced $\beta$-catenin dephosphorylation in podocytes, which later provoked elevated Snail expression upon HG stimulation ${ }^{10}$. Intriguingly, p38 could also regulate $\beta$-catenin signaling by inactivation of
GSK3 $\beta$ in mouse F9 cells $^{20}$, whereas little information is available on crosstalk between p38 and $\beta$-catenin in podocytes. Recent studies indicated that $\mathrm{C}$-terminus domain of $\beta$-catenin might constitute the minimum region necessary for $\beta$-catenin shuttling between the cytosol and nucleus ${ }^{21}$. Thus it would be of interest to further identify whether there are modifications at $\beta$ catenin C-terminus in podocytes under HG conditions. Collectively, we tested our hypothesis in the present study that podocyte-specific transgenic ablation of Rac1 might be renoprotective against podocyte damage and proteinuria via prohibiting a signaling cascade of Rac1/PAK1/ p38; and that epic activation of this signaling might also contribute to $\beta$-catenin activation and nuclear translocation in damaged podocytes both in vivo and in vitro.

\section{Results}

\section{TG mice characterization}

TG mice were characterized by immunofluorescence of the kidney cortex, and real-time PCR and western blot analysis of primary cultured podocytes. Immunofluorescence demonstrated a marked decrease in Rac1 staining in TG glomerular podocytes $(P<0.01)$ without altering synaptopodin expression $(P>0.05)$ between wild-type WT and TG mice (Fig. 1a), or podocyte numbers (Figure $S)(P>0.05)$. In primary podocytes, Rac1 expression was significantly reduced in TG mice

Table 1 Metabolic data

\begin{tabular}{|c|c|c|c|c|c|c|}
\hline & Time (week) & $n$ & WT-control & WT-STZ & TG-control & TG-STZ \\
\hline \multirow[t]{4}{*}{ Weight (g) } & 4 & 10 & $20.82 \pm 1.201$ & $17.48 \pm 2.838^{*}$ & $20.72 \pm 2.56$ & $20.11 \pm 3.048^{\#}$ \\
\hline & 6 & 11 & $21.682 \pm 1.491$ & $17.545 \pm 2.66^{*}$ & $21.855 \pm 0.856$ & $21.364 \pm 2.279^{\#}$ \\
\hline & 8 & 9 & $23.756 \pm 1.456$ & $18.256 \pm 1.978^{*}$ & $23.967 \pm 1.133$ & $21.278 \pm 2.540^{\#}$ \\
\hline & 12 & 10 & $30.51 \pm 3.975$ & $18.09 \pm 3.229^{*}$ & $30.32 \pm 3.631$ & $20.49 \pm 3.445$ \\
\hline \multirow[t]{4}{*}{ Blood glucose $(\mathrm{mmol} / \mathrm{l})$} & 4 & 10 & $7.07 \pm 1.617$ & $25.1 \pm 4.482^{*}$ & $7.14 \pm 1.977$ & $23.03 \pm 3.367$ \\
\hline & 6 & 11 & $7.2 \pm 2.106$ & $21.673 \pm 3.909^{*}$ & $7.109 \pm 1.801$ & $20.181 \pm 2.342$ \\
\hline & 8 & 9 & $7.211 \pm 2.596$ & $28.4 \pm 3.094^{*}$ & $7.356 \pm 1.882$ & $27.022 \pm 2.271$ \\
\hline & 12 & 10 & $7.79 \pm 2.424$ & $26.85 \pm 5.280^{*}$ & $7.82 \pm 1.615$ & $25.24 \pm 5.5572$ \\
\hline \multirow[t]{4}{*}{$\mathrm{SBP}(\mathrm{mmHg})$} & 4 & 10 & $107.1 \pm 3.071$ & $107.7 \pm 3.653$ & $107.8 \pm 7.7143$ & $108.1 \pm 6.4885$ \\
\hline & 6 & 11 & $107.91 \pm 4.482$ & $107.82 \pm 6.432$ & $107.82 \pm 5.419$ & $108.45 \pm 5.786$ \\
\hline & 8 & 9 & $107.89 \pm 7.424$ & $108.56 \pm 4.096$ & $107.67 \pm 5.788$ & $107.89 \pm 8.038$ \\
\hline & 12 & 10 & $108.4 \pm 6.883$ & $108.2 \pm 6.713$ & $108 \pm 1.9441$ & $107.9 \pm 4.932$ \\
\hline \multirow[t]{4}{*}{ UTP $(\mu \mathrm{g} / 24 \mathrm{~h})$} & 4 & 10 & $26.52 \pm 2.677$ & 27. $99 \pm 3.027$ & $26.65 \pm 2.521$ & $27.21 \pm 2.961$ \\
\hline & 6 & 11 & $26.255 \pm 3.191$ & $28.891 \pm 3.051$ & $26.327 \pm 2.246$ & $28.409 \pm 3.762$ \\
\hline & 8 & 9 & $26.544 \pm 2.671$ & $45.956 \pm 7.41^{*}$ & $26.311 \pm 4.378$ & $37.378 \pm 7.109^{\#}$ \\
\hline & 12 & 10 & $26.09 \pm 2.798$ & $134.48 \pm 20.549^{*}$ & $26.78 \pm 2.9139$ & $99.55 \pm 16.501^{\#}$ \\
\hline
\end{tabular}

Values represent means \pm s.d.

${ }^{*} P<0.01$ vs. control mice; ${ }^{\sharp} P<0.05$ vs. WT-STZ 


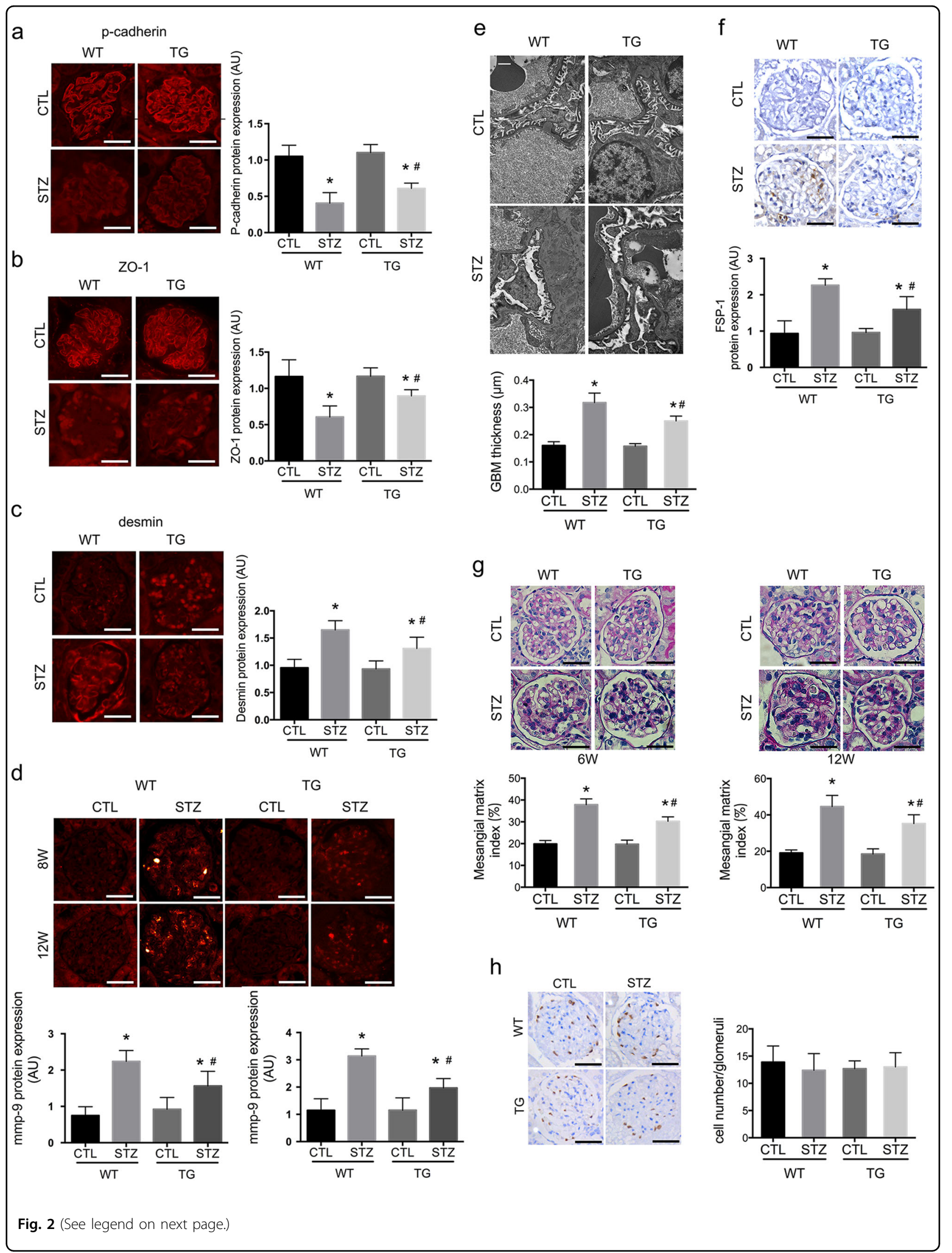




\begin{abstract}
(see figure on previous page)
Fig. 2 Effects of podocyte Rac1 deficiency on glomeruli at

12 weeks. a, b At 12 weeks, staining of podocyte-specific markers p-cadherin and ZO-1 markedly decreased in WT mice; Rac1 deletion partially restored the expression of both proteins. Values denote the mean \pm s.d.; ${ }^{*} P<0.01$ vs. $C T L,{ }^{\#} P<0.05$ vs. WT-STZ. Scale bar $=100 \mu m$. c At this time point, desmin expression was strongly stained in glomerular podocytes and parietal epithelial cells in WT diabetic mice, which was prohibited in TGSTZ mice. Values denote the mean \pm s.d.; ${ }^{*} P<0.01$ vs. CTL, ${ }^{\#} P<0.01$ vs. WT-STZ. Scale bar $=100 \mu \mathrm{m}$. d At 8 and 12 weeks, mmp-9 expression was positively stained in mesangial cells and podocytes in WT-STZ mice, which was decreased in diabetic TG mice. Values denote the mean \pm s.d.; ${ }^{*} P<$ 0.01 vs. CTL, ${ }^{*} P<0.01$ vs. WT-STZ. Scale bar $=100 \mu \mathrm{m}$. e Podocyte effacement on TEM. Podocyte FPs were found extensively effaced in WT-STZ mice, but were markedly rescued in TG-STZ counterparts. Scale bar $=1 \mu \mathrm{m}$. f De novo expression of a fibroblastic hallmark FSP-1. In CTL glomeruli, FSP-1 was negatively stained in glomerular podocytes; 12 weeks of diabetes led to de novo expression of FSP-1, which was partially diminished in TG-STZ glomeruli. Values denote the mean \pm s.d.; ${ }^{*} P<0.01$ vs. $C T L, ~ "{ }^{\#}<0.01$ vs. WT-STZ. Scale bar $=100 \mu \mathrm{m}$. g Mesangial and glomerular area by PAS staining. At 6 weeks, PAS staining showed mesangial areas were mildly expanded, with swelled endothelial cells and capillaries stenosis, in WT-STZ mice (left) and at 12 weeks, there occurred moderately mesangial proliferation and matrix accumulation, capillaries stenosis and parietal endothelial proliferation, indicative of advanced renal injury (right); these mutations were attenuated in TG diabetic mice at either time points. Values denote the mean \pm s.d. $;{ }^{*} P<0.01$ vs. $C T L,{ }^{\#} P<0.01$ vs. WT-STZ. Scale bar $=100 \mu \mathrm{m}$. $\mathbf{h}$ Podocyte numbers were determined by WT staining at 12 weeks. The graph showed no remarkable significance was calculated among four groups. Values denote the mean \pm s.d.; $P>0.05$ vs. CTL. Scale bar $=100 \mu \mathrm{m}$. AU arbitrary units, TG transgenic, WT wild type, CTL control
\end{abstract}

compared with WT mice on both protein and mRNA levels (Fig. 1b) $(P<0.05)$. There were no distinguishable differences in hair growth or appearance between WT and TG mice (Figure S); and metabolic data showed neither tendency of spontaneous proteinuria or diabetes, nor differences between two mice strains on weight and SBP levels (Table 1) $(P>0.05)$.

\section{Dedifferentiation of glomerular podocytes was found preceding the onset of proteinuria in diabetic mice}

By generating diabetes models, we showed significant differences between WT diabetic mice and their nondiabetic controls in both weight and blood glucose (BG) at $4,6,8$, and 12 weeks after the onset of diabetes (Table 1) $(P<0.05)$. In WT-STZ mice, TEM demonstrated a mild degree of foot process (FP) effusion at 6 weeks (Fig. 1c), when $\mathrm{p}$-cadherin and $\mathrm{ZO}-1$ began to show decreased staining $(P<0.01)$ (Fig. 1d, e), and a mesenchymal marker desmin presented positive staining $(P<0.01)$ (Fig. 1f). Mutations of these proteins were aggravated as diabetes progressed after 12 weeks $(P<0.01)$ (Fig. $2 \mathrm{a}-\mathrm{c})$. And mmp-9 was positively stained in glomerular podocytes and mesangial cells at 8 and 12 weeks $(P<0.01)$ (Fig. 2d). At 12 weeks, FPs were extensively effaced (Fig. 2e); and there was de novo expression of FSP-1 expression observed in podocytes $(P<0.01)$ (Fig. 2f). Moreover, proteinuria occurred at 8 weeks, succeeding theses abnormalities in glomerular podocytes, which was exacerbated at 12 weeks (Table 1$)(P<0.05)$.

\section{Podocyte-specific knockdown of Rac1 was protective against diabetic podocyte injury and proteinuria}

At 6 weeks, PAS staining showed mesangial areas mildly expanded, with swelling endothelial cells and capillaries stenosis, in WT-STZ mice (Fig. $2 \mathrm{~g}) \quad(P<0.01)$. At 12 weeks, there occurred moderately mesangial proliferation and matrix accumulation, capillaries stenosis and parietal endothelial proliferation, indicative of advanced renal injury $(P<0.01)$ (Fig. 2 g). These mutations were attenuated in TG diabetic mice at either time points $(P<0.01)$ (Fig. 2g). Rac1 deficiency partially preserved podocyte markers: staining of p-cadherin $(P<0.05)$ and ZO-1 $(P<0.01)$ were both rectified, with diminished desmin staining compared with WT-STZ mice at $6(P<$ 0.01 ) (Figs. 1d, e and 2a, b) and 12 weeks (Figs. 1f and 2c). Mmp-9 staining $(P<0.01)$ (Fig. $2 \mathrm{~d})$ as well as FSP-1 $(P<$ 0.01) (Fig. 2f) was both abolished in TG-STZ mice, in comparison with their WT diabetes littermates at the indicated time points. By TEM, we found an early alleviation of FP retraction and fusion in Rac1-depleted diabetic glomeruli at 6 weeks compared with WT-STZ group (Fig. 1c). At 12 weeks, process effacement was largely restored with ameliorated GBM in TG-STZ mice (Fig. 2e). In line with these observations, proteinuria was attenuated in TG-STZ mice at 8 and 12 weeks as soon as podocyte proteins were reinstated (Table 1$)(P<0.05)$, without alterations of BG or systolic blood pressure (SBP) (Table 1) $(P>0.05)$. The weight loss was also rescued in TG diabetic mice compared with WT hyperglycemic littermates at 4,6 , and 8 weeks (Table 1$)(P<0.05)$. Moreover, WT-1 staining revealed that podocyte numbers were not altered among four groups at 12 weeks (Fig. 2h) $(P>$ 0.05).

\section{Rac1/PAK1/p38 signaling pathway was activated in vivo and in vitro}

In primary podocytes from WT-STZ mice, Rac1 and PAK1 activities were significantly higher compared with normoglycemic controls at 6 weeks (Fig. 3a, b) $(P<0.01)$, which were both abrogated in Rac1-deficient diabetic podocytes (Fig. 3a, b) $(P<0.05)$; p38 was also heavily phosphorylated in WT diabetic podocytes (Fig. 3c) $(P<$ 


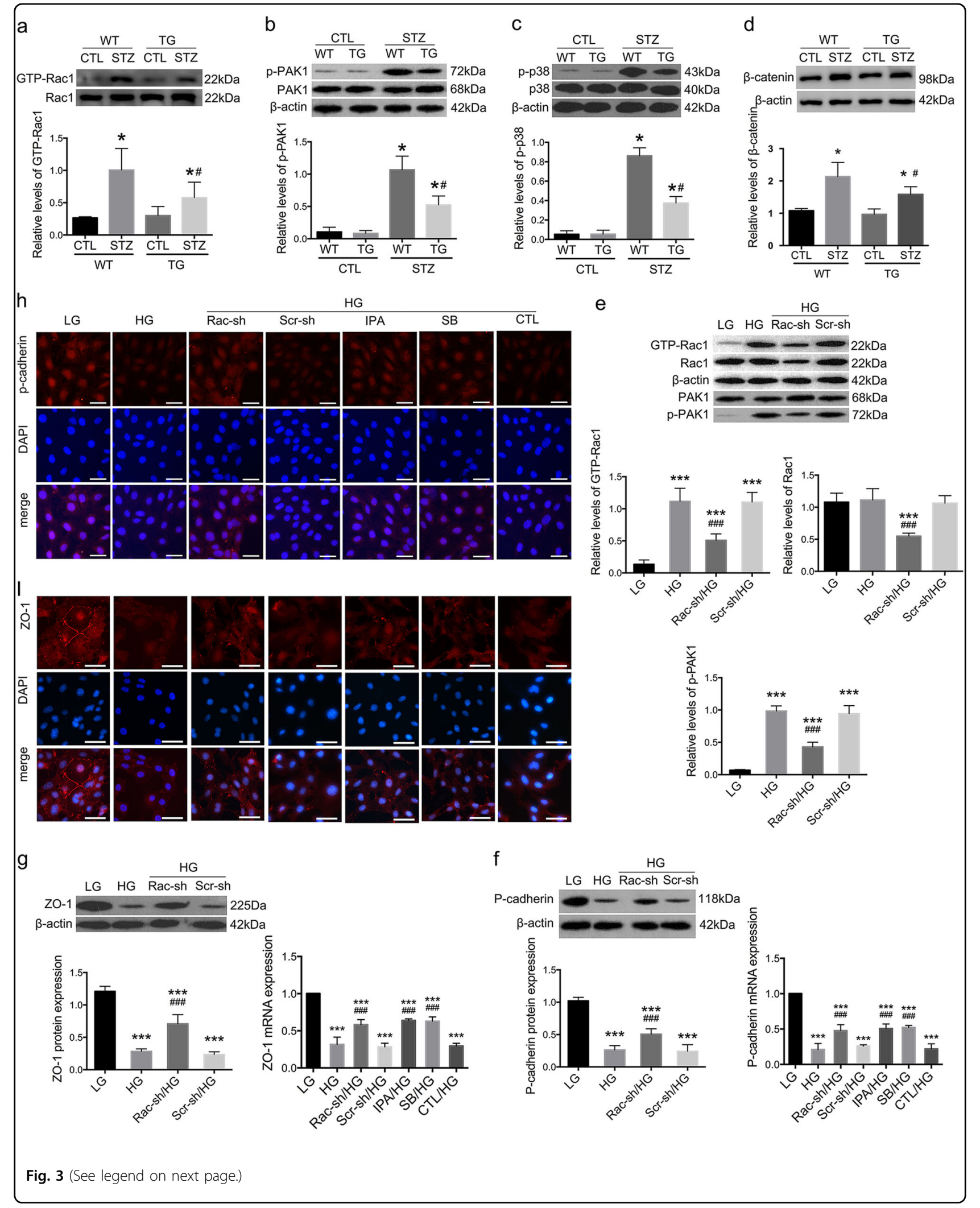


(see figure on previous page)

Fig. 3 Activation of Rac1/PAK1/p38 signaling cascade in vivo and in vitro. a-d Activation of Rac1, PAK1, p38, and $\beta$-catenin in diabetic mice. GTP-Rac1 was found significantly increased in WT diabetic mice, which was dampened by Rac1 inhibition. Western blot showed significant elevation in PAK1 and p38 phosphorylation in primary podocytes from WT diabetic mice, and overexpression of $\beta$-catenin in kidney cortices, which were partially reversed by podocyte Rac1 depletion. Values denote the mean \pm s.d. $;{ }^{*} P<0.01$ vs. $C T L,{ }^{\#} P<0.05$ vs. WT-STZ. e Alterations of Rac1 activities in cultured podocytes. HG led to marked enhancement of Rac1 activity and PAK1 phosphorylation, which were prohibited by Rac1 shRNA transfection. Total Rac1 levels were also reduced by Rac1 shRNA transfection; the lower graph depicts the relative protein levels of p-PAK1. Values denote the

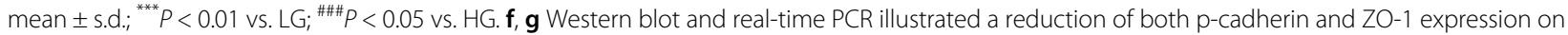
both protein and mRNA levels after HG stimulation for $48 \mathrm{~h}$. Protein levels of these two were abolished by Rac1 knockdown. PCR showed mRNA levels of p-cadherin and ZO-1 was preserved by Rac1 knockdown or IPA-3 pretreatment; SB203580 also demonstrated a reversal effect on p-cadherin and ZO-1 mRNA expression. Values denote the mean \pm s.d.; ${ }^{* * *} P<0.01$ vs. LG; ${ }^{* \# \#} P<0.05$ vs. HG. $\mathbf{h}, \mathbf{i}$ Immunofluorescence showed that $p$-cadherin was intensely stained in the cytoplasm of LG-treated podocytes. HG led to dampened staining of $\mathrm{p}$-cadherin but was reversed by Racl knockdown or pretreatment with either IPA-3 or SB203580. ZO-1 was highly expressed at cell-cell contacts under LG conditions. HG resulted to decreased ZO-1 staining in a punctate pattern; this was rectified by Rac1 shRNA transfection or pretreatment with either IPA-3 or SB203580. Cells transfected with Scramble shRNA or pretreated with DMSO were the controls. Scale bar $=200 \mu \mathrm{m}$. TG transgenic, WT wild type, CTL control, Rac-sh Rac1 shRNA, Scr-sh scramble shRNA, IPA IPA-3, SB SB203580

$0.01)$ but suppressed in TG-STZ counterparts at 6 weeks (Fig. 3c) $(P<0.05)$.

In vitro, Rac1 and PAK1 were activated in cultured podocytes after HG stimulation for $48 \mathrm{~h}$ (Fig. 3e) $(P<$ $0.01)$, despite a relatively higher apoptotic rate compared with cells treated with LG (Figure S), together with remarkable podocyte impairment. Epithelial markers p-cadherin and ZO-1 were reduced (Fig. 3f, g) $(P<0.01)$, whereas injury hallmarks $\alpha$-SMA and FSP-1 were boosted on both protein and mRNA levels (Fig. 4a, b) $(P<0.01)$. Immunofluorescence illustrated decreased staining of p-cadherin and ZO-1 (Fig. 3h, i), but intensified staining of $\alpha$-SMA and FSP-1 (Fig. 4c, d) under HG conditions. FITC-phalloidin staining showed HG for $48 \mathrm{~h}$ caused F-actin re-organization, from actin bundles along cell axis to densely interwoven filaments at cortical regions on cell periphery (Fig. 5a), together with enhanced cell motility, as was demonstrated by transwell assay (Fig. 5b) $(P<$ 0.01). Simultaneously, there was also a remarkable elevation in p38 activation stemming from HG stimulation (Fig. 4e) $(P<0.01)$.

Potential interactions between these proteins were determined. Rac1 knockdown efficiency was first confirmed (Figure $S)(P<0.05)$. Consistent with in vivo data, Rac1 shRNA transfection prevented activation of Rac1, PAK1, and p38, compared with cells without transfection (Figs. 3e and $4 \mathrm{e})(P<0.05)$; and ameliorated podocyte cell markers, evidenced by enhanced expression of $\mathrm{p}$-cadherin and ZO-1 (Fig. 3f, g), and dampened expression of $\alpha$-SMA and FSP-1 (Fig. 4a, b) $(P<0.05)$. Blunted staining of $\alpha-$ SMA and FSP-1 as well as restored podocyte hallmarks were further confirmed by Immunofluorescence (Figs. 3h, $\mathrm{i}$ and 4c, d). Next, we used two chemical inhibitors IPA-3 and SB203580 to target PAK1 and p38 respectively. PAK1 inhibition had reversal effects on both PAK1 and p38 phosphorylation (Fig. 5c, d) $(P<0.05)$, whereas hardly affected Rac1 activity (Fig. 5d) $(P>0.05)$; SB203580 showed limited impacts on Rac1 or PAK1 activation (Fig. 5d) $(P>0.05)$, albeit its inhibitory role in p38 phosphorylation driven by HG (Fig. 5c) $(P<0.05)$. Either IPA-3 or SB203580 were able to preserve expression of p-cadherin and ZO-1, and abrogate expression of dedifferentiation hallmarks desmin, $\alpha$-SMA and FSP-1 (Fig. 4f-i) $(P<0.05)$. Rac1 shRNA transfection or SB203580 pretreatment reinstated forced F-actin reassembly, as F-actin bundles at the sub-membrane region were decreased, compared with HG podocytes (Fig. 5a). Concomitantly, transwell assay presented that podocyte hypermotility driven by HG was blunted by Rac1 knockdown or p38 deactivation (Fig. 5b) $(P<0.05)$.

\section{Rac1/PAK1 signaling stabilized $\beta$-catenin by downregulating its ubiquitylation level at Ser675 in podocytes}

First we found $\beta$-catenin expression was elevated in kidney cortices of WT diabetic mice $(P<0.01)$, and reduced in Rac1-deficient hyperglycemic littermates $(P<$ 0.01) (Fig. 3d), suggesting that activated Rac1 signaling was an upstream driving force for $\beta$-catenin expression in podocytes under diabetic condition; and in HG-cultured podocytes, $\beta$-catenin nuclear translocation was provoked by HG stimulation, and was then prohibited by the blockade of Rac1/PAK1/p38 signaling pathway (Fig. 5e). By CO-IP we found PAK1 had physical interactions with $\beta$-catenin under normal culture conditions (Fig. 5f), thus we speculated Rac1 might also have physical interplays with $\beta$-catenin. Firstly, we showed $\beta$-catenin was remarkably phosphorylated at C-terminal Ser552 and Ser675 in HG-podocytes (Fig. 6a) $(\mathrm{P}<0.05)$. Next, by cotransfection of Flag- $\beta$-catenin plasmids and GFP-Rac1 mutants, we found WT-Rac1 and CA-Rac1 (V12) were able to induce $\beta$-catenin phosphorylation specifically on Ser675, whereas DN-Rac1 (N17) were not (Fig. 6b); in contrast, all three forms of Rac1 had limited phosphorylation effects on Ser552 (Fig. 6b). Moreover, treatment with proteasome inhibitor MG-132 reduced 


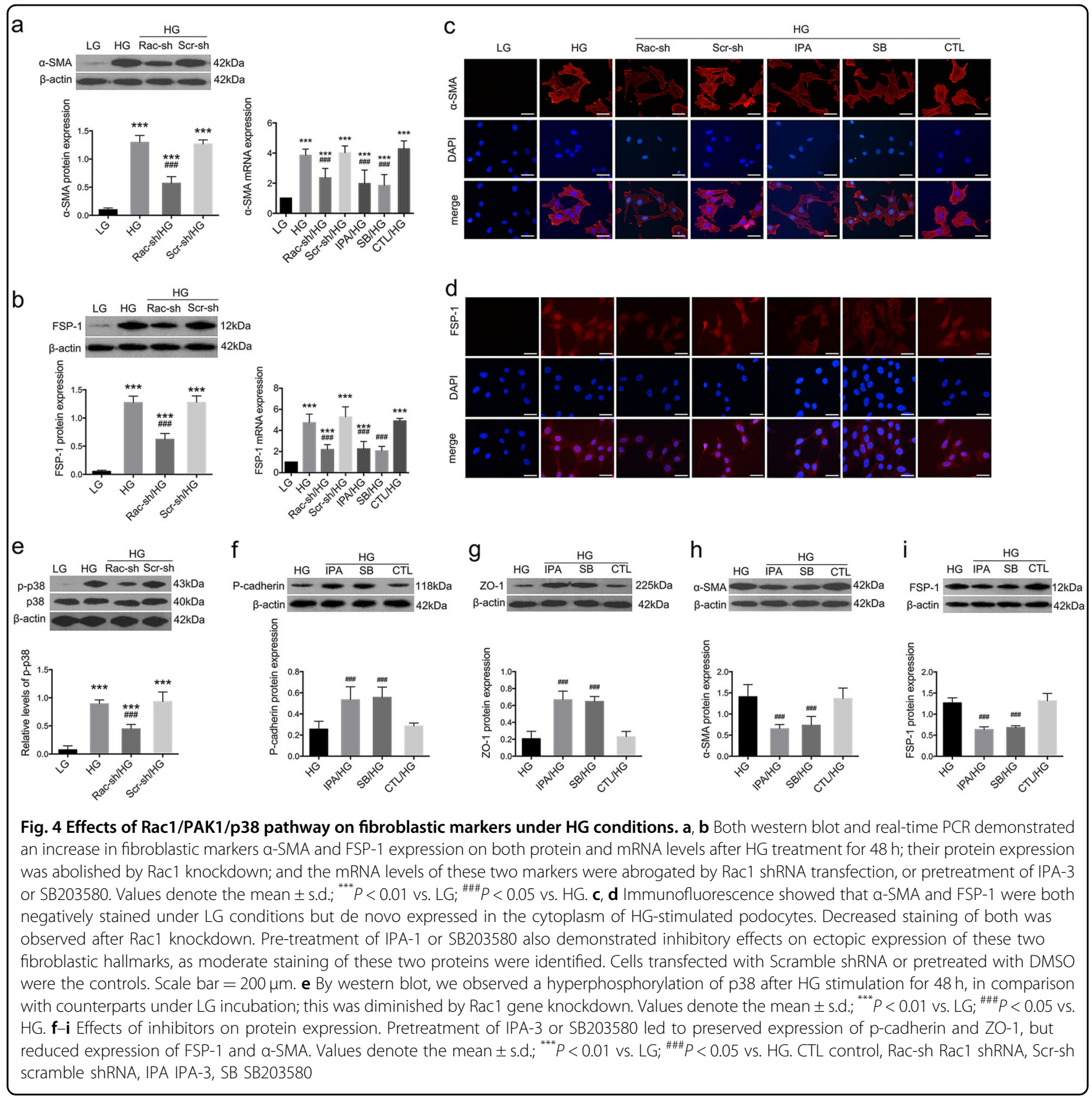

ubiquitylation level of $\beta$-catenin when Ser675 residue was markedly phosphorylated under HG conditions, compared with vehicle control (Fig. 6c).

\section{P38 accounts for HG-induced $\beta$-catenin nuclear translocation via activating MEF2c}

Also we showed a pronounced activation of MEF2c, a key transcription factor involved in controlling gene expression in several cell types including myocytes, lymphocytes, and neurons ${ }^{22}$, after HG treatment $(P<0.01)$ (Fig. 6d), which was depleted by the addition of p38 inhibitor $(P<0.05)$ (Fig. 6d), when $\beta$-catenin nuclear accumulation was also prohibited by SB203580 (Fig. 5e). Next, we performed in vitro kinase assay and showed purified MEF2c, but not $\beta$-catenin, could be directly phosphorylated by $\mathrm{p} 38$; phosphorylated MEF2c thus strongly bound to $\beta$-catenin in vitro (Fig. 6e).

\section{Discussion}

Dysfunction of glomerular podocytes has been implicated as a critical driving force for $\mathrm{DN}^{2,5}$. In the present study, we described a podocyte-specific Rac1-deficient transgenic mouse strain, which exhibited neither embryonic lethality nor spontaneous proteinuria or 


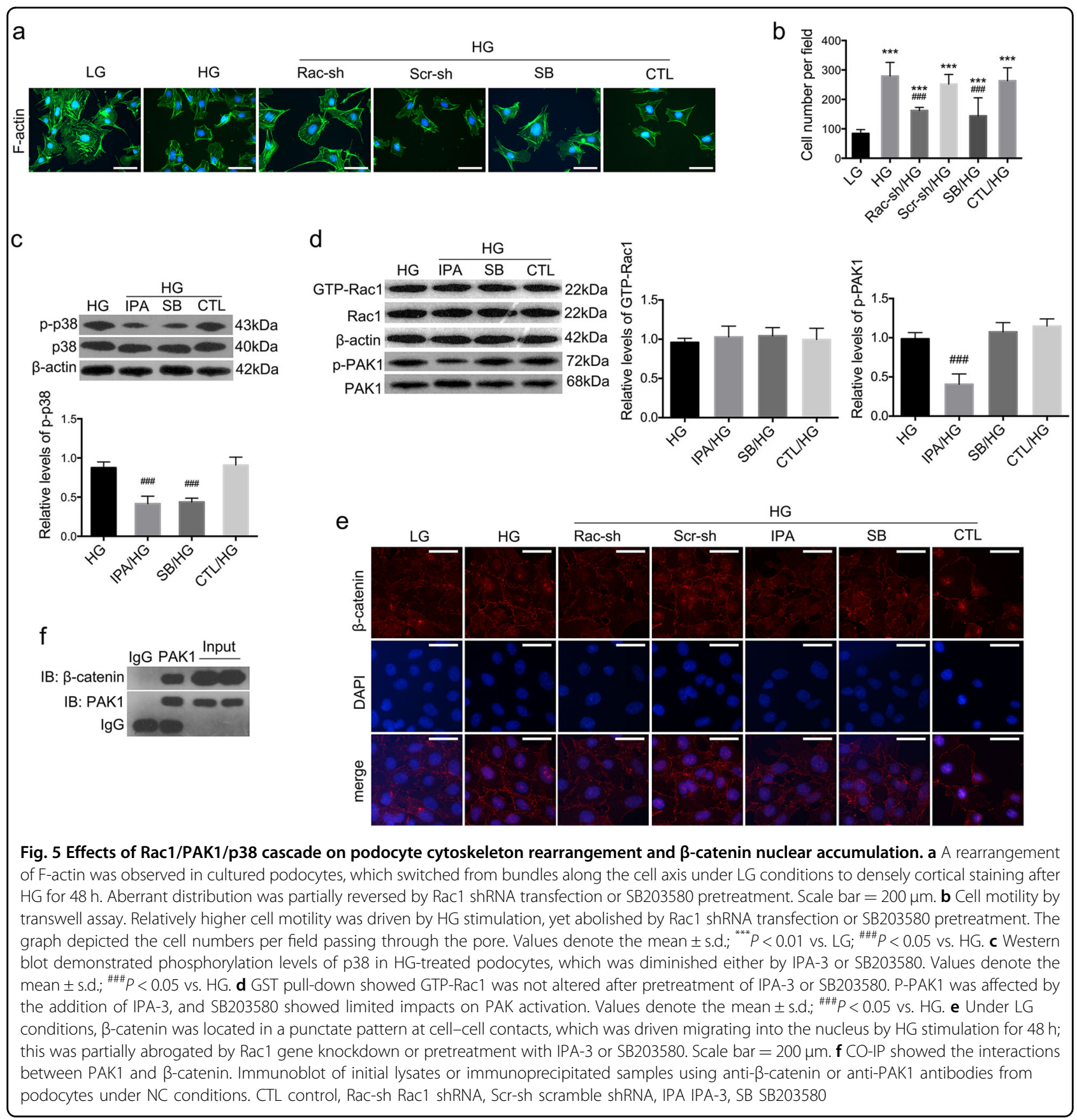

diabetes. By generating diabetes models in these TG diabetic mice as well as their littermate controls, we were able to show that podocyte-specific Rac1 depletion might be of therapeutic importance to the pathogenesis of DN.

We showed that consistent hyperglycemia caused striking structural mutations of podocyte-specific proteins $\mathrm{p}$-cadherin and ZO-1, which are also key components of the SD, and a simultaneous retraction of FPs in podocytes at as early as 6 weeks; in diabetic mice where Rac1 was depleted, these alterations were significantly restored.
Podocytes elaborate FPs surrounding glomeruli with SDs bridging interdigitations and comprising the final layer of the $\mathrm{GFB}^{23}$. SD disruption has been recognized as a key initial theme shared in various kidney diseases arising at the podocyte level ${ }^{24}$. SD generates synchronous signaling for the precise coordination of the neighboring actinbased FPs; damage to the SD was associated with uncoupled signals ${ }^{24-27}$, thus leading to a disrupted cytoskeletal structure, i.e. effaced FPs as was demonstrated here. In vitro, HG induced F-actin rearrangement 
a
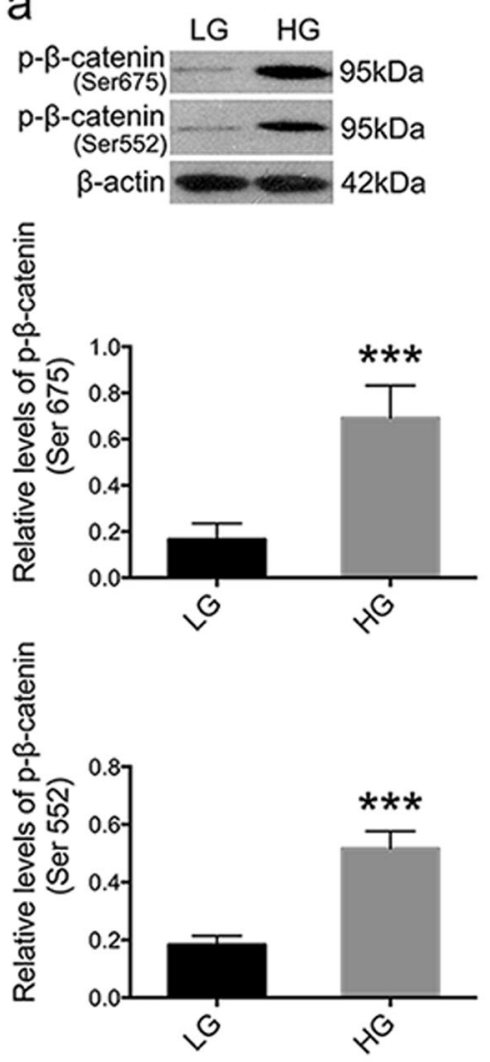

e

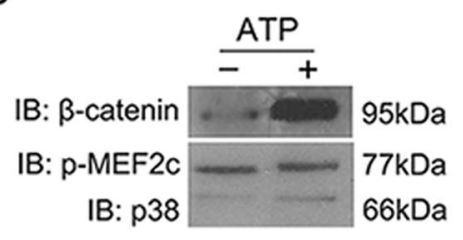

b

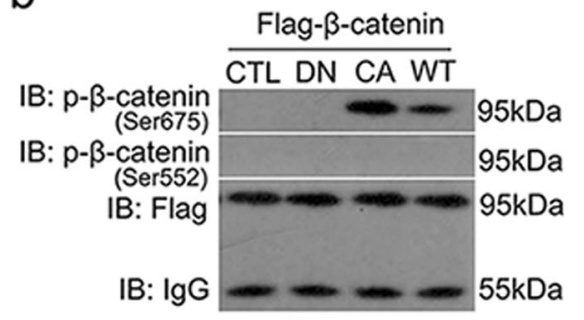

C

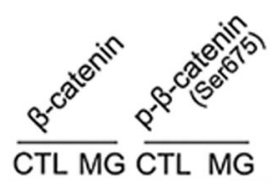

IB: Ubiquitin

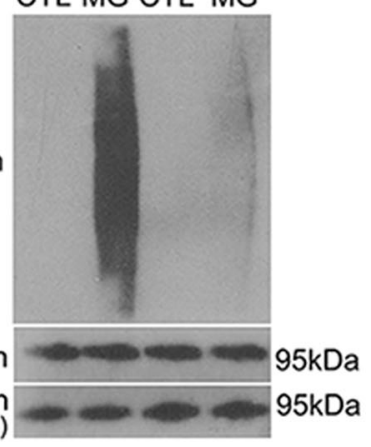
$\begin{aligned} \text { IB: } \beta \text {-catenin } & 95 \mathrm{kDa} \\ \text { B: } p-\beta \text {-catenin } & 95 \mathrm{kDa}\end{aligned}$

d HG

Fig. 6 Interplays between Rac1/PAK1/p38 and $\beta$-catenin in podocytes under culture. a Western blot demonstrated that $\beta$-catenin was phosphorylated at Ser675 and Ser552 after HG stimulation for 48 h. Values denote the mean \pm s.d. ${ }^{* * *} P<0.05$ vs. LG. b Podocytes were co-transfected with Flag- $\beta$-catenin and one of the following plasmids GFP-WT-Rac1, GFP-CA-Rac1, GFP-DN-Rac1, or an empty control vector. Cell lysates were immunoprecipitated with anti-Flag antibodies, and were subjected to immunoblot with the indicated antibodies. c HG-stimulated podocytes were treated with MG132 or DMSO for $12 \mathrm{~h}$. Cell lysates were immunoprecipitated with antibodies against total $\beta$-catenin or phospho- $\beta$-catenin (Ser675), and were subjected to immunoblot with the indicated antibodies. $\mathbf{d}$ Western blot depicted p-EMF2c was upregulated under HG conditions but was dampened by SB203580. Values denote the mean \pm s.d. ${ }^{* * *} P<0.01$ vs. LG; ${ }^{\# \# \# P} P<0.05$ vs. HG. e The interactions between p38 and $\beta$-catenin by in vitro kinase assay. Upper panel was indicative of immunoblot of $\beta$-catenin binding to MEF2c following immunoprecipitation; and the lower panel demonstrated the total phosphorylation of MEF2C after incubation with p38 and ATP. CTL control, Rac-sh Rac1 shRNA, Scr-sh scramble shRNA, IPA IPA-3, SB SB203580, DN dominant negative, CA constitutive active, WT wild type 


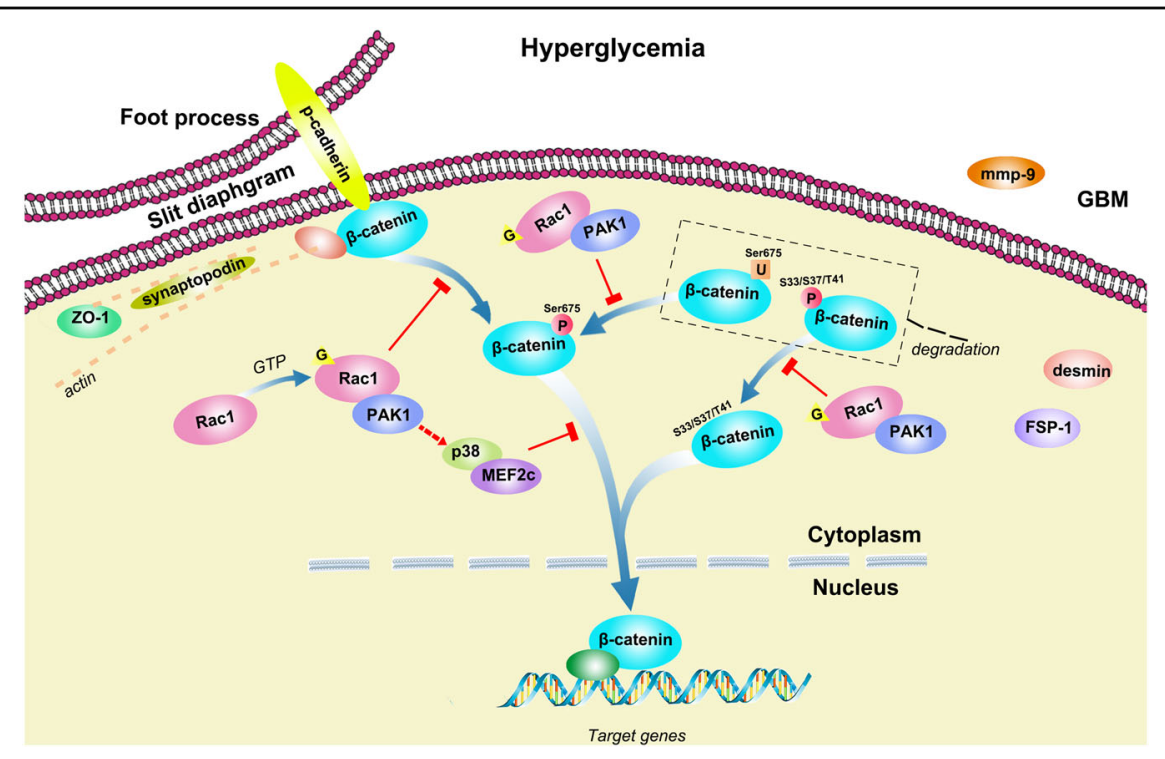

Fig. 7 Schematic illustration of Rac1/PAK1/p38 signaling in podocytes in exposure of hyperglycemia. Upon the stimulation of hyperglycemia, Rac1/PAK1 signaling was activated in podocyte; $\beta$-catenin was modified, either dephosphorylated at N-terminus or phosphorylated at C-terminus, leading to upregulated level of active $\beta$-catenin; the downstream p38 further ensured $\beta$-catenin transcriptional activities via activating MEF2c; and active $\beta$-catenin contributed to the dysregulation of a series of target genes, and imbalanced expression of SD proteins such as p-cadherin and ZO- 1

and cell hypermotility, the latter of which, is recognized as the in vitro manifestation of dynamic reorganization of the FP structure ${ }^{27}$; and these were rectified by Rac1 knockdown. These in vivo and in vitro observations demonstrated Rac1 depletion benefited diabetic podocytes through a major and early attenuation on plasticity of podocyte cytoskeleton via preserving SDs and FPs both structurally and functionally in the challenge of diabetes.

Others and we demonstrated previously that PAK1, a key modulator in regulation of actin polymerization and cell motility in mammalian fibroblasts, served as a major downstream effector of Rac1 in several cell lines including podocytes in vitro ${ }^{10,28}$. Again, we confirmed PAK1 being a downstream target for Rac1 activation in vitro and in vivo: PAK1 activation was found in podocytes of WTSTZ mice as soon as SD proteins were jeopardized, but was dampened in Rac1 TG-STZ mice. Reportedly, Rac1 contributed to LPS-mediated p38 activation in GDI $\alpha$ knockdown podocytes; ${ }^{29}$ and PAK1 could activate MAPK cascades in oncogenic transformation of a variety of cancer cells ${ }^{17}$. Likewise, we demonstrated p38 being a downstream signaling of PAK1 rather than these two proteins serving as two parallel downstream substrates in injured podocytes upon Rac1 activation, namely a signaling cascade of Rac1/PAK1/p38 in cultured podocytes. Presumably this cascade might as well exist in diabetic podocytes and play a pivotal role based on our in vivo data that simultaneous p38 activation was detected when PAK1 was also activated, and that Rac1 ablation led to reduced activation of both PAK1 and p38 in diabetic glomeruli, further showing reversal of PAK1/p38 activation was an indispensable step to reach the therapeutic effect.

Intriguingly, we found $\beta$-catenin expression and nuclear accumulation was under the modulation of Rac1/PAK1/ p38 cascade in vitro and in vivo. $\beta$-catenin is a key component of Wnt signaling pathway and active $\beta$-catenin is found in diverse kidney diseases including focal segmental glomerulosclerosis (FSGS) and $\mathrm{DN}^{19}, 30$. Excess cytoplasmic $\beta$-catenin is often targeted by a $\mathrm{N}$-terminal phosphorylation/ubiquitylation-mediated degradation system $^{31-35}$. N-terminus dephosphorylation of $\beta$-catenin is sufficient to induce stabilized $\beta$-catenin migrating into the nucleus ${ }^{10,28}$. Recent studies indicated $\beta$-catenin $C$ terminal tail constitutes the minimum region necessary for both import and export of cell nucleus in a receptorfree and energy-independent pattern ${ }^{21}$. Consistent with these studies, we demonstrated $\mathrm{C}$-terminal $\beta$-catenin was significantly phosphorylated under HG conditions, when $\beta$-catenin nuclear accumulation was marked. We further showed that Ser675 at C-terminal $\beta$-catenin was a direct phosphorylation target of Rac1 signaling: both WT- and CA-Rac1 (mimicking the pathogenic Rac1 activation upon stimuli) phosphorylated $\beta$-catenin at Ser675; this site modification was linked to obliterated ubitquitylation level of $\beta$-catenin, which meant more stabilized cytoplasmic $\beta$-catenin that could possibly enter the nucleus later on. MEF2c is a key transcription factor involved in controlling gene expression in several cell types including myocytes and neurons ${ }^{22}$. Its expression was elevated in 
cardiac tissues under experimental diabetic conditions and cardiomyocytes in exposure to high glucose ${ }^{36}$; and in a rodent model of diabetes, increased MEF2c expression was found in the retina and kidney ${ }^{37}$. Likewise, we showed the HG stimuli potentiated MEF2c activation in cultured podocytes. Also we showed that rather than directly bound to $\beta$-catenin, p38 interacted with $\beta$-catenin in a MEF2c phosphorylation-dependent manner; in other words, MEF2c could only bind to and phosphorylate $\beta$ catenin in the premise that $\mathrm{p} 38$ was activated. These aforementioned results led us to a potential mechanism that upon the stimuli of hyperglycemia, Rac1/ PAK1 signaling was activated in podocyte; $\beta$-catenin was then modified, either dephosphorylated at $\mathrm{N}$-terminus or phosphorylated at C-terminus, leading to elevated level of active $\beta$-catenin; the downstream p38 further ensured $\beta$ catenin transcriptional activities via activating MEF2c; and active $\beta$-catenin contributed to the dysregulation of a series of target genes, and imbalanced expression of SD proteins such as $\mathrm{p}$-cadherin, as reported here.

Nuclear $\beta$-catenin is responsible for the modulation of a series of EMT genes such as Twist, Snail and Slug ${ }^{38}$. EMT is a phenotypic conversion in epithelial cells, characterized by the loss of epithelial cell markers and the acquisition of fibroblastic hallmarks ${ }^{4}, 10,27,37$. We presented in vivo and in vitro the reduction in podocyte epithelial markers ( $\mathrm{p}$-cadherin and ZO-1), acquisition of fibroblastic or mesenchymal hallmarks (desmin and FSP-1), and upregulation of $\beta$-catenin expression and activation, together with a disordered cytoskeleton (effaced FPs, Factin reassembly and cell hypermotility), which were, to some extent, prevented by the blockade of Rac1/PAK1/ p38 signaling. Mature podocytes, unlike typical epithelial cells, express low levels of an intermediate filament vimentin, and retain a more "motile" cytoskeletal structure applicably assembled and disassembled to meet dynamic requirements of $\mathrm{FPs}^{39-41}$. However, we did not show other significant fibroblastic markers such as $\alpha$ SMA expressed in glomerular podocytes during the course of experimental diabetes in WT or TG mice (not shown). We hypothesized that in vivo diabetic podocytes might undergo a partial EMT or dedifferentiation process; this distinction might be attributed to far more complex circumstances in vivo instead of its direct exposure to the stimuli in vitro. Notably, there was no difference in podocyte numbers between diabetic or nondiabetic mice at this stage of diabetic nephropathy, implying that podocyte were undergoing a relatively early and adaptive mutation rather than apoptosis under such diabetic conditions, which was partially reversible, as indicated in the present study, by Rac1 signaling deficiency. The curative effect was further confirmed by the finding that the onset of proteinuria, which succeeded the podocyte damage, was as well reduced by the rectification of podocyte impairment by the blockade of Rac1 signaling. In addition, we showed Rac1 depletion had a mild attenuation on mesangial areas, implying a pleiotropic effect of podocytespecific Rac1 deficiency beyond its self-protecting mechanisms. This might be presumably attributed to reduction of mmp-9 in both podocytes and mesangial cells, which played a key role in the extracellular matrix (ECM) turnover ${ }^{42}$.

Albeit the renoprotective effects described above, we showed little impacts of Rac1 inhibition on levels of SBP or BG. Recent studies indicated a signaling cross talk between Rac1 and mineralocorticoid receptor $(M R)^{8,43}$. Since aldosterone-mineralocorticoid-receptor system has long been recognized as a pivotal player in the salt homeostasis and regulation of blood pressure ${ }^{44}$, it would be of interest to further identify whether there are any compensatory effects after Rac1 depletion. Taken together, we demonstrated podocyte-specific Rac1 depletion attenuated diabetic podocyte injury through the blockade of Rac1/PAK1/p38/ -catenin cascade, which favored DN in following aspects: (a) preserving the structure and function of SDs; (b) rectifying the sophisticated cytoskeleton and FP effacement; (c) reversing podocyte dedifferentiation; (d) reducing early mesangial injury; (e) and reducing proteinuria, without SBP or BG interference (Fig. 7). These findings provided evidence for an early therapeutic potentiality of cell-specific Rac1 deficiency in delaying the development of DN and might also shed some lights on further therapeutic strategies in proteinuric diseases.

\section{Materials and methods \\ Generation of TG mice}

The nephrin promoter-Rac1 shRNA was linearized and microinjected into C57BL/6 mouse embryos that were transferred to the oviduct of pseudopregnant recipient mice. By DNA genotyping, 3 of 19 pups were identified positive (Cyagen Biosciences, Guangzhou, China) and were crossed with $\mathrm{C} 57 \mathrm{BL} / 6$ mice to generate mice used in the present study. Forward primer: TTCGGTCGACTAGGGATAACAGG; reverse primer: TAATCCAGAGGTTGATTATCGGAA. PCR products (548 bp) were analyzed by agarose gel electrophoresis. The littermates lacking the transgene were control wild type (WT) mice.

\section{Diabetes models}

All animal studies were carried out with the review and approval of the animal care and use committee of Shandong University. At 8 weeks of age, 160 male WT and TG mice randomly allocated into two groups, either intraperitoneally injected with STZ (Sigma-Aldrich, St Louis, MO) dissolved in citrate buffer $(50 \mathrm{mg} / \mathrm{kg}$ for 5 consecutive days) ( $\mathrm{pH} 4.5)$ or citrate buffer alone. 
Hyperglycemic state was monitored and maintained on a standard rodent diet with water ad libitum. SBP was examined by tail-cuff manometry (BP-2000, Visitech Systems, NC). Urine was collected for determination of urinary protein when mice were weighed and placed in metabolic cages for $24 \mathrm{~h}$. At the end of $4,6,8$, and 12 weeks, mice were sacrificed and kidneys were harvested for following experiments.

\section{Transmission electron microscopy}

Small pieces of renal cortex were fixed in glutaraldehyde and osmic acid, dehydrated with an ethanol gradient, soaked in ethoxyline resin overnight, and mounted at $60^{\circ}$ $\mathrm{C}$ for $48 \mathrm{~h}$. Ultrathin sections were cut and viewed under H-7500 TEM (Hitachi, Tokyo, Japan).

\section{Cell culture}

Isolation of glomeruli was done by three-step sieving $(250,100$, and $70 \mu \mathrm{m})$ of kidney cortices ${ }^{45}$. Isolated glomeruli were maintained on type I collagen in RPMI 1640 $(10 \% \mathrm{FBS}, 100 \mathrm{U} / \mathrm{ml}$ penicillin and $100 \mathrm{mg} / \mathrm{ml}$ streptomycin) (Life Technologies, CA, USA). After 7 days, glomeruli were removed and cells were trypsinized and passed through a $40-\mu \mathrm{m}$-pore size sieve to remove mesangial and endothelial cells ${ }^{46}$. Podocytes identity was then done by synaptopodin and WT-1 immunofluorescence. Conditionally immortalized mouse podocytes were kindly provided by Professor Peter Mundel (Massachusetts General Hospital, Boston, MA, USA) via Professor Jie Ding (Peking University). Podocytes were cultured as described previously ${ }^{10,46}$.

\section{Transfection and RNA interference}

Rac1 shRNA 5'-GACGTGTTCTTAATTTGCTT-3', a scrambled shRNA $5^{\prime}$-GCGCGCTTTGTAGGATTCG-3', Flag- $\beta$-catenin vectors, GFP-WT-Rac1, GFP-constitutive active (CA)-Rac1 (CA) (V12), GFP-dominant negative (DN)-Rac1 (N17), and an empty vector, were from Cyagen Biosciences (Guangzhou, China). Transfection was performed $\left(1 \times 10^{6}\right.$ cells/well $)$ with indicated plasmids using Lipofectamine3000 reagent (Invitrogen, Life Technologies Corporation). After Rac1 shRNA incubation, podocytes were either treated with low glucose (LG) $(5.6 \mathrm{mM})$ or HG $(30 \mathrm{mM})$ for an additional $48 \mathrm{~h}$. Cells transfected with scrambled shRNA and incubated with HG for $48 \mathrm{~h}$ were controls.

\section{Measurement of Rac1 activity}

Rac1 activation was assessed using a pull-down assay kit according to the manufacturer's instructions (\#80501, NewEast Biosciences, PA, USA). Briefly, cells were harvested and then lysed with assay/lysis buffer containing protease inhibitors on ice. The cell lysates were then centrifuged and protein content was estimated using the Bio-Rad protein assay. To analyze active Rac1 levels, each sample was adjust the volume of to $1 \mathrm{ml}$ with $1 \times$ assay/lysis Buffer Lysate and incubated with $1 \mu \mathrm{l}$ anti-active Rac1 monoclonal antibody, bound with protein A/G agarose beads for $1 \mathrm{~h}$ at $4{ }^{\circ} \mathrm{C}$. The GTPbound forms of Rac1 in the pull-down samples were precipitated, run in a 10\% SDS-PAGE gel and subjected to western blot.

\section{Co-immunoprecipitation}

Co-IP was performed using Universal Magnetic Co-IP Kit according to the manufacturer's instructions (\#54002, Active Motif).

\section{In vitro kinase assay}

Recombinant GST-p38 and GST-myocyte-specific enhancer factor 2C (MEF2c) (Abnova, Heidelberg, Germany) were incubated in kinase buffer for $3 \mathrm{~h}$ at $30^{\circ} \mathrm{C}$ with or without ATP addition (NEB, Ipswich, USA), followed by $6 \times$ His- $\beta$-catenin (Cyagen Biosciences, Guangzhou, China), glutathione-agarose beads (Thermo, IL, USA), and NP-40 lysis buffer (Solarbio, Beijing, China). Beads were heated, run in SDS-PAGE gel, and subjected to western blot.

\section{Western blot}

The protocol was described previously ${ }^{10}$. Primary antibodies used were as follows: p-cadherin (Santa Cruz, CA, USA), FSP-1, $\alpha$-SMA, $\beta$-catenin, mmp-9, Rac1 (Abcam, MA, USA); phospho- $\beta$-catenin (Ser552), phospho- $\beta$ catenin (Ser675), phospho-p38 (Thr180/Tyr182), p38, PAK1 and phospho-PAK1 (Thr423) (Cell Signaling Technology, MA, USA), $\beta$-actin (Proteintech, IL, USA), ZO-1 (Thermo Fisher Scientific, IL, USA) and desmin (Immunoway). Bands were detected using ECL system (Amersham Biosciences, Piscataway, NJ, USA) and BioRad electrophoresis image analyzer (Bio-Rad, Hercules, CA, USA).

\section{Real-time reverse transcriptase PCR}

Total RNA extraction and reverse transcription, and amplification were described as previously ${ }^{10}$. Primers were from Sangon Biotech (Shanghai, China). Sequences were designed as follows (Table 2).

\section{Histology, immunohistochemistry, and immunofluorescence}

Kidney sections were stained via PAS method. Podocyte counts were determined by WT-1 staining. For immunofluorescence, kidney sections or fixed cells were immunostained with primary antibodies at $4{ }^{\circ} \mathrm{C}$ overnight and Dylight 594- or 488-conjugated IgG the next day; FITC-phalloidin $(50 \mu \mathrm{g} / \mathrm{ml})$ was used for F-actin staining. All analyses were performed in a blinded fashion. Images 
Table 2 Primers for real-time PCR

\begin{tabular}{lll}
\hline Gene & Primer & Sequence \\
\hline P-cadherin & Sense & 5'-GTTGAGCCGCAGAAGTATGA-3' \\
& Antisense & 5'-GAGTGGTGATGGTGAAATGGT-3' \\
ZO-1 & Sense & 5'-GAGCTACGCTTGCCACACTGT-3' \\
& Antisense & 5'-TCGGATCTCCAGGAAGACACT-3' \\
a-SMA & Sense & 5'-TGTGTGAAGAGGAAGACAGCA-3' \\
& Antisense & 5'-TCCAACCATTACTCCCTGATG-3' \\
FSP-1 & Sense & 5'-TGTGTCCACCTTCCACAAATAC-3' \\
& Antisense & 5'-ACTTCATTGTCCC TGTTGCTGT-3' \\
B-actin & Sense & 5'-AAGACGAGGAGGAACTGAAC-3' \\
& Antisense & 5'-CAAATCGGA CAACAAGACG-3' \\
Rac1 & Sense & 5'-GTAAAACCTGCCTGCTCATCA-3' \\
& Antisense & 5'-GGGACGCAATCTGTCATAATC-3' \\
\hline
\end{tabular}

were captured on an inverted phase/fluorescence microscope (Leica Microsystems GmbH, Wetzlar, Germany).

\section{Transwell migration assay}

Transwell cell-culture inserts $(8 \mu \mathrm{m})$ (Corning, MA, USA) were placed in RPMI1640 (10\% FBS) in lower compartment. Podocytes were seeded in upper chambers $\left(1 \times 10^{4} / \mathrm{ml}\right)$, which were allowed to attach at $37^{\circ} \mathrm{C}$ for 48 h. Non-migratory cells were removed; migrated cells were stained with haematoxylin. Cell number in the center of a membrane (one field) was counted using phase contrast microscopy (Leica Microsystems $\mathrm{GmbH}$ ). Data presented denote the mean \pm s.d. of three independent experiments.

\section{Statistics}

Experiments were performed at least three times. Values were reported as mean \pm s.d. Data were analyzed using SPSS 19.0 software. Statistical significance was assessed using Student's $t$-test, and one-way ANOVA and LSD- $t$ test; and $P<0.05$ were considered to be statistically significant.

\section{Acknowledgements}

This work was funded by National Natural Science Foundation of China (Grant 81400732, 81370834, and 30971381), Shandong Young Scientists Award Fund (2010BSB14076), and Chinese Society of Nephrology Scientific Fund (14050480585). All authors approved the final version of the manuscript.

\section{Author details}

${ }^{1}$ Department of Nephrology, Shandong Provincial Hospital Affiliated to Shandong University, Jinan, China. ${ }^{2}$ Department of Obstetrics and Gynecology, The Second Hospital of Shandong University, Jinan, China. ${ }^{3}$ Institute of Basic Medicine, Shandong Academy of Medical Sciences, Jinan, China. ${ }^{4}$ Department of Pathology, School of Medicine, Shandong University, Jinan, China. ${ }^{5}$ Department of Internal Medicine Clinic, Shandong Provincial Hospital Affiliated to Shandong University, Jinan, China

\section{Conflict of interest}

The authors declare that they have no conflict of interest.

\section{Publisher's note}

Springer Nature remains neutral with regard to jurisdictional claims in published maps and institutional affiliations.

Supplementary Information accompanies this paper at https://doi.org/ 10.1038/s41419-018-0353-z.

Received: 19 November 2017 Revised: 22 January 2018 Accepted: 23 January 2018

Published online: 01 March 2018

\section{References}

1. Kanwar, Y. S., Sun, L., Xie, P., Liu, F. Y. \& Chen, S. A glimpse of various pathogenetic mechanisms of diabetic nephropathy. Annu. Rev. Pathol. 6 395-423 (2011)

2. Reiser, J. \& Sever, S. Podocyte biology and pathogenesis of kidney disease. Annu. Rev. Med 64, 357-366 (2013).

3. Brinkkoetter, P. T., Ising, C. \& Benzing, T. The role of the podocyte in albumin filtration. Nat. Rev. Nephrol. 9, 328-336 (2013).

4. Reidy, K. \& Suszta, K. Epithelial-mesenchymal transition and podocyte loss in diabetic kidney disease. Am. J. Kidney Dis. 54, 590-593 (2009).

5. Bid, H. K., Roberts, R. D., Manchanda, P. K. \& Houghton, P. J. RAC1: an emerging therapeutic option for targeting cancer angiogenesis and metastasis. Mol. Cancer Ther. 12, 1925-1934 (2013).

6. Marei, H. \& Malliri, A. Rac1 in human diseases: the therapeutic potential of targeting Rac1 signaling regulatory mechanisms. Small GTPases 21, 1-25 (2016).

7. Kowluru, R. A. et al. TIAM1-RAC1 signalling axis-mediated activation of NADPH oxidase-2 initiates mitochondrial damage in the development of diabetic retinopathy. Diabetologia 57, 1047-1056 (2014).

8. Kawarazaki, W. et al. Angiotensin II- and salt-induced kidney injury through Rac1-mediated mineralocorticoid receptoractivation. J. Am. Soc. Nephrol. 23 997-1007 (2012).

9. Kolavennu, V., Zeng, L., Peng, H., Wang, Y. \& Danesh, F. R. Targeting of RhoA ROCK signaling ameliorates progression of diabetic nephropathy independent ofglucose control. Diabetes 57, 714-723 (2008).

10. LV, Z. et al. Rac1/PAK1 signaling promotes epithelial-mesenchymal transition of podocytes in vitro via triggering $\beta$-catenin transcriptional activity under high glucose conditions. Int. J. Biochem. Cell Biol. 45, 255-264 (2013).

11. Nagase, M., Kurihara, H., Aiba, A., Young, M. J. \& Sakai, T. Deletion of Rac1GTPase in the myeloid lineage protects against inflammation-mediated kidney injury in mice. PLOS ONE 11, e0150886 (2016).

12. Sugihara, $\mathrm{K}$. et al. Racl is required for the formation of three germ layers during gastrulation. Oncogene 17, 3427-3433 (1998).

13. Stambe, C., Atkins, R. C., Hill, P. A. \& Nikolic-Paterson, D. J. Activation and cellular localization of the p38 and JNK MAPK pathways in rat crescenticglomerulonephritis. Kidney Int. 64, 2121-2132 (2003).

14. Tang, S. C. \& Lai, K. N. The pathogenic role of the renal proximal tubular cell in diabetic nephropathy. Nephrol. Dial. Transplant. 27, 3049-3056 (2012).

15. Martineau, L. C., McVeigh, L. I., Jasmin, B. J. \& Kennedy, C. R. p38 MAP kinase mediates mechanically induced COX-2 and PG EP4 receptor expression in podocytes:implications for the actin cytoskeleton. Am. J. Physiol. Ren. Physiol. 286, F693-F701 (2004)

16. $\mathrm{Hu}, \mathrm{M}$. et al. FAK contributes to proteinuria in hypercholesterolaemic rats and modulates podocyte F-actin re-organization via activating p38 in response to ox-LDL. J. Cell Mol. Med 21, 552-567 (2017).

17. Wang, R. A., Zhang, H., Balasenthil, S., Medina, D. \& Kumar, R. PAK1 hyperactivation is sufficient for mammary gland tumor formation. Oncogene $\mathbf{2 5}$, 2931-2936 (2006)

18. Dechert, M. A., Holder, J. M. \& Gerthoffer, W. T. p21-activated kinase 1 participates in tracheal smooth muscle cell migration by signaling to p38 Mapk. Am. J. Physiol. Cell Physiol. 281, C123-C132 (2001).

19. Wu, X. et al. Rac1 activation controls nuclear localization of beta-catenin during canonical Wnt signaling. Cell 133, 340-353 (2008). 
20. Bikkavilli, R. K., Feigin, M. E. \& Malbon, C. C. p38 mitogen-activated protein kinase regulates canonical Wnt-beta-catenin signaling by inactivation ofGSK3beta. J. Cell Sci. 121, 3598-3607 (2008).

21. Koike, $\mathbf{M}$. et al. beta-Catenin shows an overlapping sequence requirement but distinct molecular interactions for itsbidirectional passage through nuclear pores. J. Biol. Chem. 279, 34038-34047 (2004).

22. Ma, K., Chan, J. K., Zhu, G. \& Wu, Z. Myocyte enhancer factor 2 acetylation by p300 enhances its DNA binding activity, transcriptional activity, and myogenic differentiation. Mol. Cell Biol. 25, 3575-3582 (2005).

23. Holthöfer, H. Molecular architecture of the glomerular slit diaphragm: lessons learnt for a better understanding ofdisease pathogenesis. Nephrol. Dial. Transplant. 22, 2124-2128 (2007).

24. Durvasula, R. V. \& Shankland, S. J. Podocyte injury and targeting therapy: an update. Curr. Opin. Nephrol. Hypertens. 15, 1-7 (2006).

25. Grahammer, F., Schell, C. \& Huber, T. B. The podocyte slit diaphragm--from a thin grey line to a complex signalling hub. Nat. Rev. Nephrol. 9, 587-598 (2013).

26. Greka, A. \& Mundel, P. Cell biology and pathology of podocytes. Annu. Rev. Physiol. 74, 299-323 (2012)

27. Kistler, A. D., Altintas, M. M. \& Reiser, J. Podocyte GTPases regulate kidney filter dynamics. Kidney Int 81, 1053-1055 (2012)

28. Sells, M. A., Boyd, J. T. \& Chernoff, J. p21-activated kinase 1 (Pak1) regulates cell motility in mammalian fibroblasts. J. Cell Biol. 145, 837-849 (1999).

29. Robins, R., Baldwin, C., Aoudjit, L., Gupta, I. R. \& Takano, T. Loss of Rho-GDla sensitizes podocytes to lipopolysaccharide-mediated injury. Am. J. Physiol. Ren. Physiol. 308, F1207-F1216 (2015)

30. Dai, C. et al. Wnt/beta-catenin signaling promotes podocyte dysfunction and albuminuria. J. Am. Soc. Nephrol. 20, 1997-2008 (2009).

31. Olivier-Van, S. S. et al. O-GlcNAcylation stabilizes $\beta$-catenin through direct competition with phosphorylation at threonine 41. FASEB J. 28, 3325-3338 (2014).

32. Kato, $H$. et al. Wnt/ $\beta$-catenin pathway in podocytes integrates cell adhesion, differentiation, and survival. J. Biol. Chem. 286, 26003-26015 (2011).

33. McClelland Descalzo, D. L. et al. Glucose-induced oxidative stress reduces proliferation in embryonic stem cells via FOXO3A/ 3 -catenin-dependent transcription ofp21(cip1). Stem Cell Rep. 7, 55-68 (2016).
34. Sastre-Perona, A. \& Santisteban, P. Wnt-independent role of $\beta$-catenin in thyroid cell proliferation and differentiation. Mol. Endocrinol. 28, 681-695 (2014).

35. Li, C. C., Le, K., Kato, J., Moss, J. \& Vaughan, M. Enhancement of $\beta$-catenin activity by BIG1 plus BIG2 via Arf activation and CAMP signals. Proc. Natl Acad. Sci. USA 113, 5946-59451 (2016).

36. Feng, B., Chen, S., George, B., Feng, Q. \& Chakrabarti, S. miR133a regulates cardiomyocyte hypertrophy in diabetes. Diabetes Metab. Res. Rev. 26, 40-49 (2010).

37. Xu, B., Chiu, J., Feng, B., Chen, S. \& Chakrabarti, S. PARP activation and the alteration of vasoactive factors and extracellular matrix protein in retina and kidney in diabetes. Diabetes Metab. Res. Rev. 24, 404-412 (2008).

38. Miller, R. K., Hong, J. Y., Muñoz, W. A. \& McCrea, P. D. Beta-catenin versus the other armadillo catenins: assessing our current view of canonical Wnt signaling. Prog. Mol. Biol. Transl. Sci. 116, 387-407 (2013).

39. Liu, Y. New insights into epithelial-mesenchymal transition in kidney fibrosis. J. Am. Soc. Nephrol. 21, 212-222 (2010).

40. Chuang, P. Y. \& He, J. C. Signaling in regulation of podocyte phenotypes. Nephron Physiol. 111, p9-p15 (2009).

41. May, C. J., Saleem, M. \& Welsh, G. I. Podocyte dedifferentiation: a specialized process for a specialized cell. Front. Endocrinol. 5, 148 (2014).

42. Li, Y. et al. Epithelial-to-mesenchymal transition is a potential pathway leading to podocyte dysfunction andproteinuria. Am. J. Pathol. 172, 299-308 (2008).

43. Shibata, S. et al. Modification of mineralocorticoid receptor function by Rac1 GTPase: implication in proteinuric kidneydisease. Nat. Med. 14, 1370-1376 (2008).

44. Nagase, M. \& Fujita, T. Role of Rac1-mineralocorticoid-receptor signalling in renal and cardiac disease. Nat. Rev. Nephrol. 9, 86-98 (2013).

45. Schiwek, D. et al. Stable expression of nephrin and localization to cell-cell contacts in novel murine podocyte cell lines. Kidney Int. 66, 91-101 (2004).

46. Mundel, P. et al. Rearrangements of the cytoskeleton and cell contacts induce process formation during differentiation of conditionally immortalized mouse podocyte cell lines. Exp. Cell Res. 36, 248-258 (1997). 